\title{
Analysis of Vasopressin-Induced Transepithelial Water Transport by Confocal and Electron Microscopy Methods
}

\author{
A. N. Gorshkov, E. S. Snigirevskaya, and Ya. Yu. Komissarchik \\ Institute of Cytology, Russian Academy of Sciences, St. Petersburg, Russia; \\ e-mail: angorsh@yahoo.com
}

DOI: $10.1134 / \mathrm{S} 1990747809030222$

It is known that water transport across the vasopressin-sensitive epithelia is mediated by water channel proteins, aquaporins. The binding of arginin-vasopressin (AVP) to V2-receptors launches an adenylyl cyclase-signaling pathway, which is terminated by translocation of aquaporin 2 from the pool of cytoplasmic vesicles to the apical plasma membrane. In our work, to analyze the structural bases of water transport, we utilized two model objects: an amphibian urinary bladder and MDCK cells grown on the polyester membrane filters with the pore diameter of $0.4 \mu \mathrm{m}$.

It is shown by freeze-fracture that under the action of AVP, water channels appear on the PF-surface of apical membrane of amphibian urinary bladder epithelial cells and are visualized as massive intramembranous particles aggregates.

In two model objects studied electron microscopy revealed similar ultrastructural alterations, which affect predominantly cytoskeleton and Golgi complex elements. Under the AVP action, actin cytoskeleton of epi- theliocytes is depolymerized significantly, which is a prerequisite of the physical contact of vesicles carrying the water channels to the apical membrane. Analogous results were obtained by rhodamine-phalloidin labeling of F-actin in epitheliocytes. Using confocal microscopy, in a series of optical sections it is shown that AVP caused depolymerization of F-actin associated with stress-fibers and of apical submembranous actin, while actin associated with intercellular contacts did not undergo notable rearrangements.

AVP also induced fragmentation of the Golgi complex and swelling in its cisternae. This effect of inclusion of water into the vacuoles, which are the Golgi complex derivates, represents a mechanism of adaptation of AVP-sensitive epithelia cells to the hypotonic environment and, apparently, serves as a complementary way of the cellular volume regulation.

The work was supported by the Russian Foundation for Basic Research (project no. 06-04-48281-a). 